\title{
Physiological control systems for high-quality interdisciplinary researches
}

\author{
Levente Kovács \\ Research and Innovation Center of Óbuda University, Physiological Controls Group \\ Obuda University, Budapest, Hungary \\ kovacs.levente@nik.uni-obuda.hu
}

\begin{abstract}
The aim of the Physiological Controls Group of Obuda University is to investigate pathophysiological systems and develop mathematical models in order to create automatic control algorithms or model-based treatment protocols enhancing personalized healthcare possibilities. The presentation deals with the given problem starting with a short introduction on the control of diabetes and then focuses on tumor control aspects. The latter will be presented from the innovative aspect of combining control engineering with antiangiogenic targeted molecular therapy, rewarded recently by the EU's European Research Council with the highly competitive ERC StG grant.
\end{abstract}

Physiological modelling and control represents the basic topic of biomedical engineering, the mostly developing engineering field nowadays (reflected in the recent STEM $^{1}$ job projections as well). All of the thirteen other subdisciplines of biomedical engineering lies on the modelling and control aspects. Basically in all these cases the two basic challenges to be faced are connected to understand the physiological processes by describing them in mathematical models, and to find adequate methods to identify the required signals from the measured ones. Consequently, this approach opens the way to personalized healthcare by giving the possibility to support medical devices for specific diseases or ease everyday life healthcare monitoring (i.e. by smartphone applications). As a result, it has a direct connection to the nowadays spreading cyber-medical systems' concept as well.

The Physiological Controls Group ${ }^{2}$ (PhysCon) of the Obuda University was created in 2013 with the aim of assembling the physiological and pathophysiological research activities at Obuda University, in addtion creating a direct connection to medical partners as well. The target researches of PhysCon are connected to diabetes (the socalled artificial pancreas problem), tumor control, biostatistical analysis of public health problems and control solutions for hemodialysis machines.

The background of the PhysCon researches lies on the robust control engineering approaches developed for diabetes. The Artificial Pancreas concept connects sensorics (continuous glucose monitors and insulin pumps) with the development of a control algorithm to treat diabetic patients. The idea was developed for type 1 diabetes, where the complete insufficiency of the human body in producing insulin gives a direct connection to drug delivery systems using control engineering

${ }^{1}$ STEM - acronym of Science, Technology, Engineering, and Mathematics fields.

${ }^{2}$ http://physcon.uni-obuda.hu/?q=en applications. A robust control concept was developed at PhysCon and tested by in-silico measurements using real patient data of the insulin pump centers of the Hungarian Artificial Pancreas Working Group.

In the meantime, the idea was transplanted to cancer researches, in particular to the antiangiogenic targeted molecular therapy (ATMT) approach. Arising in the last decade, the aim of ATMT is to stop the formation of new blood vessels and in this way to cease the growth of the tumor. However, the corresponding drugs are very expensive; moreover, in high dose can cause serious and dangerous side effects. The empirically determined medical protocols gave us a direct connection to the physiological modeling and control idea: reduce and keep the tumor in a minimal and safe volume by automated control algorithms, and in this way stop tumor growth, maintaining it in a minimal, "tamed" form.

The result of the research could be a personalized healthcare approach for drug-delivery in cancer, improving the patients' quality of life (QoL), optimizing drug infusion and minimizing treatment costs. Due to the heterogeneous nature of the patients, the robust control approach is envisaged again. For this, we have to reidentify the tumor growth model based on the newest medical knowledge, to demonstrate the applicability and cost-optimality of a quasi-continuous low-dosage therapy protocol in medical practice and finally to design adequate control algorithm for the problem.

The above-mentioned goals are the basis of the recently rewarded European Research Council's (ERC) StG grant. The ERC grants represent the excellence individual grants of the EU, providing attractive and long-term funding for researchers who demonstrated scientific excellence. The aim of this highly competitive research scheme is to promote ground-breaking, high-gain/high-risk research ideas in order to boost Europe research competence, but also to support principal investigators at the career stage to consolidate their research teams.

The aim of the plenary talk is dual: to give a general overview of the interdisciplinary possibilities of the physiological control field (focusing specially on cancer researches), but also to give a (subjective) example on how a research problem could be rewarded as an excellence research idea.

\section{ACKNOWLEDGEMENT}

The work is supported by the European Research Council Starting Grant ERC-StG 679681. 
L. Kovács • Physiological Control Systems for High-Quality Interdisciplinary Researches 